\title{
EDITORIAL
}

\section{Are statins beneficial for viral pneumonia?}

\author{
Angela R. Boyd* and Eric M. Mortensen\#
}

$\mathbf{R}$ espiratory infections, primarily pneumonia and influenza, continue to be the leading infectious cause of death in the developed world [1,2]. Despite the introduction of new classes of antibiotics there has been little progress in improving pneumonia-related outcomes since the widespread introduction of antibiotics in the 1940s [3]. In addition, there has been little progress on agents to potentially blunt mortality from another influenza pandemic [4]. Despite great hope for agents, such as drotrecogin alfa and corticosteroids, no new classes of medications have been conclusively demonstrated to improve clinical outcomes for viral or bacterial pneumonias. Therefore, additional treatments are critically needed to improve clinical outcomes for patients with pneumonia.

Recently, epidemiological studies of HMG-CoA reductase inhibitors ("statins") have suggested that patients receiving statins have decreased incidence of serious infections, or for those hospitalised with viral or bacterial infections, including pneumonia, have improved clinical outcomes [5-9]. Other studies have found that statins attenuate the systemic inflammatory response [10-13]. In addition, statins have been demonstrated to have protective endothelial effects, influence inflammatory cell signalling, directly affect T-cell activity, and influence the nitric oxide balance to promote haemodynamic stability [14-16].

While awaiting adequately powered randomised control trials to examine the effects of statins on pneumonia outcomes, experimental studies have investigated the effect of statins on airway infection and inflammatory diseases using in vitro cell culture and in vivo animal models. In this issue of the European Respiratory Journal, LEE et al. [17] provide novel mechanistic insights into how statins modulate the antiviral inflammatory response. Importantly, this study is one of the few that demonstrate that statins modulate the antiviral response in human bronchial and epithelial cells, the first line of defence against invading pathogens.

Using primary normal human bronchial epithelial cells (NHBE) and a human type II pneumocyte cell line A549 they demonstrate that statins attenuate viral dsRNA-induced AKT phosphorylation, STAT3 activation and the subsequent production of RANTES (regulated on activation of normal T-cell, expressed and secreted). RANTES is a CC-chemokine that was

*University of Texas Health Science Center at San Antonio, San Antonio, TX, and \#VA North Texas Health Care System and University of Texas Southwestern Medical Center, Dallas, TX, USA.

CORRESPONDENCE: E.M. Mortensen, Dallas VA Medical Center, General Internal Medicine (111E), 4500 South Lancaster, Dallas, TX 75216, USA. E-mail: Eric.Mortensen@UTSouthwestern.edu originally identified to be chemotactic to T-cells but is now recognised to stimulate the influx of numerous inflammatory cells, including monocytes, eosinophils and neutrophils [18]. The question remains: how might these effects translate in vivo? To answer this question they developed a dsRNAinduced pneumonia mouse model. In previous animal models, statins have been delivered parenterally or orally; however, it is unclear whether systemic administration of statins modulates the inflammatory response of the lung epithelium. Therefore, statins were delivered by the intranasal route, which allowed direct contact with the respiratory epithelium. Using immunohistochemistry, they demonstrated that administration of statins decreased STAT3 and RANTES expression in airway epithelia compared to controls. In addition to decreasing RANTES production, statins also attenuated the pro-inflammatory cytokine response and decreased neutrophil influx, findings consistent with previously published studies, including our own [19-21].

The majority of published experimental reports describe beneficial effects associated with statin prophylaxis with regards to airway inflammation in animal models of chronic asthma, chronic obstructive pulmonary disease and bacterial pneumonia [22-24]. However, few studies have examined how statins modulate the antiviral inflammatory response in the lungs. For example, statins have been found to reduce lipopolysaccharide-induced lung inflammation in human volunteers and decrease airway hyperreactivity in a mouse model of chronic asthma [25-27]. However, LEE et al. [17] did not extend their studies to examine outcomes following viral pneumonia. A recent mouse model of statins and influenza A pneumonia found no difference in survival, airway inflammation or cellular infiltration [28]. However, rosuvastatin was administered in food only 3 days prior to infection with influenza A. It is likely that the timing and duration of statin use prior to infection has a significant impact on the antiinflammatory and immunomodulatory properties of statins. In fact, the majority of studies using animal models that report reduced lung inflammation administer statins for weeks prior to infection. Thus, whether statins protect against viral infections remains unclear, although some benefit seems to be likely for prolonged users. Further animal and human research is needed before definite conclusions can be made.

Along such lines, a recent retrospective study of 3043 patients, using data from the Centers for Disease Control and Prevention's Emerging Infections Program influenza hospitalisation surveillance system, reported that statin use is associated with reduced mortality during and after hospitalisation for influenza virus infection (adjusted OR 0.59, 95\% CI 
0.38-0.92) [9]. Although this study was not the first to report reduced risk of influenza death among statin users [8, 29], it was the first to limit their analyses to laboratory confirmed influenza A infection. Unfortunately, this study did not include data on the causes of death in this population and so the question remains: how are statins reducing mortality? A major complication following viral pneumonia is secondary bacterial pneumonia. As the overwhelming inflammation and resolution of inflammation is thought to contribute to the occurrence of secondary bacterial infection [30], it remains to be seen whether the anti-inflammatory effects of statins during viral pneumonia, as reported by LEE et al. [17], might reduce the incidence or severity of secondary bacterial pneumonia.

Unfortunately it is unclear if the effects seen by LEE et al. [17] would be seen in those who take statins chronically for primary or secondary cardioprotection, especially as the intranasal route of administration is not currently available for routine patient care. However, it is clear that additional research into the underlying mechanisms, and potential beneficial effects, of statins for both viral and bacterial infections is needed. Although animal and human research to date is promising it is still unclear if these medications are truly beneficial and whether acute initiation of statins will ever be recommended at the time of diagnosis for those with viral and/or bacterial infections.

\section{SUPPORT STATEMENT}

This material is the result of work supported with resources and facilities of the VA North Texas Health Care System. The views expressed in this article are those of the author and do not necessarily represent the views of the Department of Veterans Affairs.

\section{STATEMENT OF INTEREST}

None declared.

\section{REFERENCES}

1 Kung HC, Hoyert DL, Xu JQ, et al. Deaths: Final data for 2005. National Vital Statisitcs Reports; vol 56, no 10. Hyattsville, National Center for Health Statistics, 2008.

2 World Health Organization. The Global Burden of Disease: 2004 Update. Geneva, WHO Press, 2008.

3 Gilbert K, Fine MJ. Assessing prognosis and predicting patient outcomes in community-acquired pneumonia. Semin Respir Infect 1994; 9: 140-152.

4 Fedson DS. Confronting the next influenza pandemic with antiinflammatory and immunomodulatory agents: why they are needed and how they might work. Influenza Other Respi Viruses 2009; 3: 129-142.

5 Nakashima B, Restrepo MI, Anzueto A, et al. The potential role of statins in pneumonia. Curr Respir Med Rev 2010; 6: 155-161.

6 Chopra V, Rogers MA, Buist M, et al. Is statin use associated with reduced mortality after pneumonia? A systematic review and meta-analysis. Am J Med 2012; 125: 1111-1123.

7 Novack V, MacFadyen J, Malhotra A, et al. The effect of rosuvastatin on incident pneumonia: results from the JUPITER trial. CMAJ 2012; 184: E367-E372.

8 Kwong JC, Li P, Redelmeier DA. Influenza morbidity and mortality in elderly patients receiving statins: a cohort study. PLOS ONE 2009; 4: e8087.

9 Vandermeer ML, Thomas AR, Kamimoto L, et al. Association between use of statins and mortality among patients hospitalized with laboratory-confirmed influenza virus infections: a multistate study. J Infect Dis 2012; 205: 13-19.

10 de Bont N, Netea MG, Rovers C, et al. LPS-induced cytokine production and expression of LPS-receptors by peripheral blood mononuclear cells of patients with familial hypercholesterolemia and the effect of HMG-CoA reductase inhibitors. Atherosclerosis 1998; 139: 147-152.

11 Jialal I, Stein D, Balis D, et al. Effect of hydroxymethyl glutaryl coenzyme a reductase inhibitor therapy on high sensitive Creactive protein levels. Circulation 2001; 103: 1933-1935.

12 Musial J, Undas A, Gajewski P, et al. Anti-inflammatory effects of simvastatin in subjects with hypercholesterolemia. Int J Cardiol 2001; 77: 247-253.

13 Ridker PM, Rifai N, Pfeffer MA, et al. Inflammation, pravastatin, and the risk of coronary events after myocardial infarction in patients with average cholesterol levels. Cholesterol and Recurrent Events (CARE) Investigators. Circulation 1998; 98: 839-844.

14 Almog Y. Statins, inflammation, and sepsis: hypothesis. Chest 2003; 124: 740-743.

15 Hothersall E, McSharry C, Thomson NC. Potential therapeutic role for statins in respiratory disease. Thorax 2006; 61: 729-734.

16 Terblanche M, Almog Y, Rosenson RS, et al. Statins and sepsis: multiple modifications at multiple levels. Lancet Infect Dis 2007; 7: 358-368.

17 Lee CS, Yi EH, Lee JK, et al. Simvastatin suppresses RANTESmediated neutrophilia in polyinosinic-polycytidylic acid-induced pneumonia. Eur Respir J 2013; 41: 1147-1156.

18 Levy JA. The unexpected pleiotropic activities of RANTES. J Immunol 2009; 182: 3945-3946.

19 Benati D, Ferro M, Savino MT, et al. Opposite effects of simvastatin on the bactericidal and inflammatory response of macrophages to opsonized S. aureus. J Leukoc Biol 2010; 87: 433-442.

20 Rosch JW, Boyd AR, Hinojosa E, et al. Statins protect against fulminant pneumococcal infection and cytolysin toxicity in a mouse model of sickle cell disease. J Clin Invest 2010; 120: 627-635.

21 Fessler MB, Young SK, Jeyaseelan S, et al. A role for hydroxymethylglutaryl coenzyme a reductase in pulmonary inflammation and host defense. Am J Respir Crit Care Med 2005; 171: 606-615.

22 Kim DY, Ryu SY, Lim JE, et al. Anti-inflammatory mechanism of simvastatin in mouse allergic asthma model. Eur J Pharmacol 2007; 557: 76-86.

23 Zeki AA, Franzi L, Last J, et al. Simvastatin inhibits airway hyperreactivity: implications for the mevalonate pathway and beyond. Am J Respir Crit Care Med 2009; 180: 731-740.

24 Boyd AR, Hinojosa C, Rodriguez PJ, et al. Impact of oral simvastatin therapy on acute lung injury in mice during pneumococcal pneumonia. BMC Microbiol 2012; 12: 73.

25 Methe H, Kim JO, Kofler S, et al. Statins decrease Toll-like receptor 4 expression and downstream signaling in human CD14+ monocytes. Arterioscler Thromb Vasc Biol 2005; 25: 1439-1445.

26 Novack V, Eisinger M, Frenkel A, et al. The effects of statin therapy on inflammatory cytokines in patients with bacterial infections: a randomized double-blind placebo controlled clinical trial. Intensive Care Med 2009; 35: 1255-1260.

27 Shyamsundar M, McKeown ST, O'Kane CM, et al. Simvastatin decreases lipopolysaccharide-induced pulmonary inflammation in healthy volunteers. Am J Respir Crit Care Med 2009; 179: 1107-1114.

28 Radigan KA, Urich D, Misharin AV, et al. The effect of rosuvastatin in a murine model of influenza A infection. PLOS ONE 2012; 7: e35788.

29 Frost FJ, Petersen H, Tollestrup K, et al. Influenza and COPD mortality protection as pleiotropic, dose-dependent effects of statins. Chest 2007; 131: 1006-1012.

30 van der Sluijs KF, van der Poll T, Lutter R, et al. Bench-to-bedside review: bacterial pneumonia with influenza - pathogenesis and clinical implications. Crit Care 2010; 14: 219. 